\title{
Development and Deployment of Automated Machine Learning Detection in Electron Microcopy Experiments
}

Kevin G. Field ${ }^{1}$, Ryan Jacobs ${ }^{2}$, Mingen Shen ${ }^{2}$, Matthew Lynch ${ }^{1}$, Priyam Patki ${ }^{1}$, Christopher Field ${ }^{3}$ and Dane Morgan $^{4}$

${ }^{1}$ University of Michigan, United States, ${ }^{2}$ University of Wisconsin, United States, ${ }^{3}$ Theia Scientific, LLC, Arlington, Virginia, United States, ${ }^{4}$ University of Wisconsin Madison, United States

Electron microscopy (EM) is only a decade away from reaching its centennial birthday and, during its maturation, has become the de facto method for evaluating materials evolution for a range of environments. The look, feel, and operation of electron microscopes have changed significantly since the birth of the field, but the way we analyze the images generated from EM has only come from one generational leap - digitization. Digitization has eliminated the bottleneck associated with the critical activity of generating, organizing, and storing electron images, but one still remains after nearly ninety years: identification, classification, and quantification of the features present in an analog or digital EM image. The EM community has identified this issue and is now leveraging modern, automated feature detection algorithms, such as those built on large, deep convolution neural networks (CNNs) [1-9]. The use of CNNs may provide for a second generational leap automation. Ultimately, issues have arisen limiting the community's ability to arrive at this leap. The first issue is the lack of substantial, unbiased ground truth labeled images for training CNNs, a second is developing and vetting CNNs for real-time detection, and a third issue is deploying the trained CNNs at the point-of-acquisition in an easy to use and consumable digital format.

Here, we demonstrate on-going pursuits toward providing automation in electron microscopy images using CNNs. The first pursuit is the application of a lightweight, reduced ordered transmission electron microscopy image simulation method to generate large amounts of "synthetic" data for training common CNN architectures. Specifically, we have developed a Python-based image generation algorithm that uses simplified phase-contrast models to simulate features of interest in either an under- or over-focused bright field Transmission Electron Microscopy (BF-TEM) condition. The output of this physics-based simulation is then integrated with experimentally obtained background images to create novel "synthetic" images with automatically labeled (via both bounding box and pixel segmentation) features. Figure 1 shows the developed method's output where cavities (e.g., open volume in the microstructure - spherical in nature due to energy minimization) generated under neutron irradiation in a 316 stainless steel are imaged in BF-TEM conditions. Note, for the example provided in Figure 1a, $30 \%$ of the cavities in the image are simulated while the remaining fraction is original to the background image. We will show that our developed method enables rapid generation of unbiased ground truth labeled images that can serve as surrogate and/or supplemental data for the training and evaluation of CNN-based automated feature detection algorithms.

The second is the exploration of using and deploying CNNs, specifically the You Only Look Once (YOLO) real-time object detection algorithm [10], to perform real-time inference during in-situ microscopy experiments. Specifically, we focus on detecting small defect clusters and dislocation loops generated from an in-situ TEM ion irradiation experiment. In this configuration, an electron transparent specimen is observed via TEM with the electron beam $\sim 90^{\circ}$ to the specimens. Also, an ion beam, or multiple ion beams, is brought at an acute angle to the specimen to create displacements and damage, leading to the nucleation and growth of the defect clusters. Recent advancements using the YOLO algorithm have shown human-like performance levels (e.g., F1 scores >0.80) in characterizing these induced in-situ experiments - an example of the output for a single frame of an in-situ video is shown in Figure 1b. The values of such a method are readily observed, as in post-analysis, dynamic events such as nucleation, growth, and coalescence can be mapped both temporally (or via irradiation dose) and spatially. We will show the temporal and spatial mapping for a variety 
of in-situ experiments as well as highlight on-going developments to deploy the YOLO-based method for realtime detection at the point-of-acquisition.
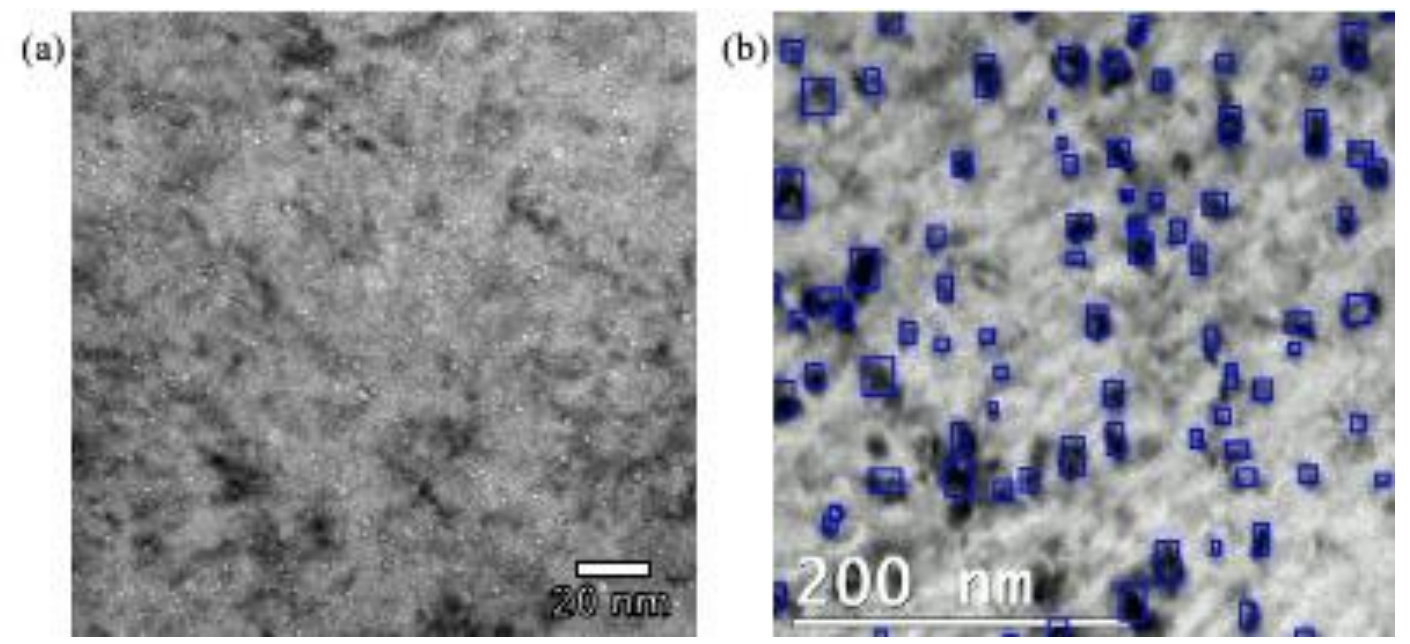

Figure 1. (a) BF-TEM image where $30 \%$ of cavities (bright, near-circular contrast) are generated synthetically using a simplified phase contrast method. (b) BF-TEM image showing bounding box detection (blue contrast) using YOLO v1.3 on defect clusters (black contrast) generated via in-situ ion irradiation.

\section{References}

[1] W. Li, K.G. Field, D. Morgan, Automated defect analysis in electron microscopic images, Npj Comput. Mater. 4 (2018) 36. doi:10.1038/s41524-018-0093-8.

[2] G. Roberts, S.Y. Haile, R. Sainju, D.J. Edwards, B. Hutchinson, Y. Zhu, Deep Learning for Semantic Segmentation of Defects in Advanced STEM Images of Steels, Sci. Rep. 9 (2019) 12744. doi:10.1038/s41598019-49105-0.

[3] C.M. Anderson, J. Klein, H. Rajakumar, C.D. Judge, LK. B, Automated Classification of Helium Ingress in Irradiated X-750, ArXiv:1912.04252. (2019) 1-7.

[4] G.D. Förster, A. Castan, A. Loiseau, J. Nelayah, D. Alloyeau, F. Fossard, C. Bichara, H. Amara, A deep learning approach for determining the chiral indices of carbon nanotubes from high-resolution transmission electron microscopy images, Carbon N. Y. 169 (2020) 465-474. doi:10.1016/j.carbon.2020.06.086.

[5] C. Zhang, J. Feng, L.R. DaCosta, P.M. Voyles, Atomic resolution convergent beam electron diffraction analysis using convolutional neural networks, Ultramicroscopy. $210 \quad$ (2020) 112921. doi:10.1016/j.ultramic.2019.112921.

[6] Z.X. Yu, S.C. Wei, J.W. Zhang, B. Wang, Y.J. Wang, Y. Liang, H.L. Tian, High-throughput, algorithmic determination of pore parameters from electron microscopy, Comput. Mater. Sci. 171 (2020) 109216. doi:10.1016/j.commatsci.2019.109216.

[7] X. Li, O. Dyck, R.R. Unocic, A. V. Ievlev, S. Jesse, S. V. Kalinin, Statistical learning of governing equations of dynamics from in-situ electron microscopy imaging data, Mater. Des. 195 (2020) 108973. doi:10.1016/j.matdes.2020.108973.

[8] M. Ziatdinov, S. Jesse, B.G. Sumpter, S. V Kalinin, O. Dyck, Tracking atomic structure evolution during directed electron beam induced Si-atom motion in graphene via deep machine learning, Nanotechnology. (2020). doi:10.1088/1361-6528/abb8a6.

[9] C.K. Groschner, C. Choi, M.C. Scott, Methodologies for Successful Segmentation of HRTEM Images via Neural Network, (2020).

[10] J. Redmon, A. Farhadi, YOLOv3: An Incremental Improvement, (2018). 